\title{
Signaled reinforcement effects on fixed-interval performance of the rat
}

\author{
SADAHIKO NAKAJIMA and KATSUYA KITAGUCHI \\ Kwansei Gakuin University, Nishinomiya, Japan
}

\begin{abstract}
In three experiments using fixed-interval schedules with a 500-msec delay of reinforcement, rats receiving a localized light signal during the delay leverpressed more slowly than rats trained without the signal. In Experiment 1 these groups showed no differences in temporal patterning of responding, but in the remaining two experiments the signaled rats showed better patterning than the unsignaled rats. In Experiments 2 and 3 rats receiving a diffuse tone signal during the delay instead of a light pressed more rather than less rapidly than the unsignaled group. Their patterning was better than that of the unsignaled rats in one of these experiments. Several explanations for both the attenuation and enhancing effects of signaling reward are discussed.
\end{abstract}

Animals trained on variable-interval (VI) schedules of briefly delayed reinforcement respond more slowly when an explicit signal stimulus fills the delay. At first, this phenomenon was interpreted as evidence that the signal overshadows an instrumental response or restricts the development of response-reinforcer association, resulting in the attenuation of the rate of responding (Dickinson, Peters, \& Shechter, 1984; Hall, 1982; Hall, Channell, \& Pearce, 1981; Pearce \& Hall, 1978; St. Claire-Smith, 1979, 1987). However, the incremental effect of the signal on responding under variable-ratio (VR) schedules (Reed \& Hall, 1988; Reed, Schachtman, \& Hall, 1988a, 1988b; Schachtman \& Reed, 1990) contradicted the overshadowing interpretation and led to the claim that the signal facilitates acquisition of a specific unit of behavior preceding reinforcement: slow responding on VI schedules and rapid responding on VR schedules. Several experiments have supported this hypothesis (Reed, 1989a, 1989b; Reed \& Hall, 1988, 1989; Reed, Schachtman, \& Hall, 1991; Reed, Schachtman, \& Rawlins, 1992).

The claim that the signal facilitates the unit of behavior preceding reinforcement is inconsistent with the fact that rate of responding is attenuated by the signal under

This research was supported by a Fellowship of the Japan Society for the Promotion of Science (JSPS) to S.N., who is now at the Department of Psychology, University of Pennsylvania. The research was conducted with a Grant-in-Aid for Scientific Research, Ministry of Education, Science and Culture of Japan, to S.N. as a PD Fellow of the JSPS (H5-0808). S.N. also received a JSPS Postdoctral Fellowship for Research Abroad when the final manuscript was being written. We would like to thank H. Imada for many helpful discussions and encouragement, and P. Reed, R. M. Tarpy, and W. Timberlake for valuable comments on the manuscript. Portions of the data were presented at the 54th annual meeting of the Japanese Society for Animal Psychology, held at Hokkaido in August 1994. Correspondence should be addressed to S. Nakajima, Department of Psychology, University of Pennsylvania, 3815 Walnut St., Philadelphia, PA 19104 (e-mail: nakajima@) cattell.psych.upenn.edu).

-Accepted by previous editor, Vincent M. LoLordo a fixed-interval (FI) schedule (Tarpy, Roberts, Lea, \& Midgley, 1984). On FI schedules, rate of responding is low after the reinforcement and increases prior to the next reinforcement, resulting in the well-known scallop or break-and-run pattern (Dews, 1978; Ferster \& Skinner, 1957; Schneider, 1969). Thus, if the unit of behavior to be facilitated is the rapid responding in the later portion of the interreinforcement interval, the signal should enhance, rather than attenuate, the rate of responding.

According to Tarpy, Lea, and Midgley (1983), the rate of interval responding is a product of competition between two predictive elements, the response and the temporal interval offset and the signal enhances the salience of the latter. This hypothesis appropriately predicts response attenuation on both FI and VI schedules. On interval schedules, the response is followed by the reinforcement only after the interval. If the signal enhances the salience of the temporal interval offset, it should attenuate the rate of responding. Application of this hypothesis to FI responding also implies a change of the response pattern in the signaled condition: sharper scallops and/or longer postreinforcement pauses (time to the breakpoint to run). This is because an increased salience of the interval offset should lead to better temporal discrimination. Unfortunately, this prediction was not examined in the study of Tarpy et al. (1984).

In the series of experiments reported here, we directly investigated the effects of a signal on the rate and pattern of FI performance of rats. In Experiment 1 we tested the hypothesis of Tarpy et al. (1983) using a localized light signal. In Experiments 2 and 3, we compared the effects of localized light versus diffuse tone as signals of reinforcement.

\section{EXPERIMENT 1}

In this experiment, we compared two groups of rats trained on an FI 60-sec schedule of briefly delayed reinforcement. A light signal filled the delay for one group, 
and there was no signal for the other. Tarpy et al. (1984) examined the effect of a light signal on FI responding using a between-subjects design with a yoked-signal control group. Rats in the yoked group received the signal when their paired master rats received the signal and the reinforcement. This procedure, however, might have introduced some artifacts because a stimulus in the interval affects the rate and pattern of FI responding (e.g., Eckerman \& McGourty, 1969; Farmer \& Schoenfeld, 1966a, 1966b; Ferster \& Skinner, 1957; Kendall, 1972; Segal, 1962). Thus, we used as an appropriate control the unsignaled group instead of the yoked one. Other details of the procedure, including the nature of the signal, FI values, and the number of reinforcements per session, were similar to those of Tarpy et al. (1984).

\section{Method}

Subjects. Subjects were 12 experimentally naive male Wistar rats housed individually in wire-mesh cages with free access to water. Prior to the experiment they were reduced to $80 \%$ of their free-feeding weights (range, 307-361 g) for pretraining. Then they were maintained at $85 \%$ of their free-feeding weights by supplementary feeding after each session. Experimental sessions were conducted 7 days a week.

Apparatus. A $30.5-\mathrm{cm}$ long, $24.7-\mathrm{cm}$ wide, and $27-\mathrm{cm}$ high chamber was housed in a ventilated sound-attenuating shell with an observation window. The chamber was made of stainless steel front and back panels, two clear acrylic side walls, a clear acrylic ceiling, and a grid floor consisting of stainless steel rods $(0.5 \mathrm{~cm}$ in diameter) spaced $1.3 \mathrm{~cm}$ apart. A 3-cm wide, 1-cm thick stainless steel lever protruded $2.6 \mathrm{~cm}$ from the front panel, and presses of the lever $(.10 \mathrm{~N})$ were detected by a microswitch. The edge of the lever was $2.4 \mathrm{~cm}$ from the left wall and $4 \mathrm{~cm}$ above the floor. A bulb $(24 \mathrm{~V} \mathrm{dc}, 0.11 \mathrm{~A})$ with a clear jeweled cover was located on the front panel $5 \mathrm{~cm}$ above the right edge of the lever and was used as a signal light. The front panel had another bulb $(24 \mathrm{~V} \mathrm{dc}$, $0.11 \mathrm{~A}$ ) with a white jeweled cover, $25 \mathrm{~cm}$ above the floor, and it was used as a center houselight. A $1-\mathrm{cm}$ wide food cup protruded $1.8 \mathrm{~cm}$ from the center of the front panel and it was $2 \mathrm{~cm}$ above the floor. A pellet dispenser delivered a $45-\mathrm{mg}$ pellet (Muromachi Kikai Co., Japan) into the food cup with a click ( $92 \mathrm{~dB}$, Scale C, against $70-\mathrm{dB}$ background ventilation fan noise). Experimental events were controlled and recorded by a microcomputer with an interface board.

Procedure. Acclimation to the chamber was conducted on Day 1. After the food cup was filled with six pellets, rats were exposed to the chamber for $30 \mathrm{~min}$ during which the houselight was on and the lever was inoperative. During Days 2 and 3 , the rats were trained to press the lever and eat the pellets. A pellet was delivered automatically by the dispenser every $60 \mathrm{sec}$. In addition, every press on the lever immediately produced one pellet and reset the 60 -sec timer for the free pellets. Before the sessions, the food cup was filled with pellets and the lever was baited with crushed pellets. The session began with the onset of the houselight and it was terminated $1 \mathrm{sec}$ after the 40 th pellet was delivered. An additional hand-shaping session was conducted on Day 3 for rats that had failed to press the lever spontaneously. The window of the sound-attenuating shell, which was usually closed, was open for the hand shaping.

Then two groups of 6 rats were matched to the extent possible for performance during pretraining, the order of running on daily sessions, and body weights. Group Unsig received leverpress training on FI schedules with a 500-msec delay of reinforcement: The first response after the fixed interval following the previous reinforcer (or from the onset of the session for the first interval) initiated the delay and then produced the reinforcer. Group Sig received the same training except that the delay was filled with a 500 -msec light signal. The session began with the onset of the houselight and terminated $1 \mathrm{sec}$ after the final reinforcer. The interval value was $15 \mathrm{sec}$ on Day 4 and $30 \mathrm{sec}$ on Day 5 , and the number of reinforcements was 60 and 50, respectively. During the remaining 16 days (Days 6-21), thirty 60-sec intervals were programmed.

Measures. Each session duration consisted of 30 (or 60 and 50 on Days 4 and 5, respectively) intervals and reinforcement delays and accumulation of waste time. Each waste time was the time from the end of the interval to the response that initiated the delay. Each interval was split into 10 consecutive time "segments," and the number of responses during each segment was accumulated for a whole session. In addition, the number of responses during the delay was recorded. Thus the total number of responses in a given session was the sum of the interval responses, the delay responses, and the 30 (or 60 and 50) responses that initiated the delay.

The recording was not in interval-by-interval fashion, so that only the data accumulated over the entire session were available. From these accumulated records, the following measures were calculated for each session.

1. Response rate: The sum of the total number of the interval responses and the responses that initiated the delay was divided by the sum of the total interval duration and the cumulative waste time. This is the rate except during the delay period. We also calculated the overall rate (i.e., the total number of responses during the session divided by the total session duration) and the interval rate (i.e., the sum of the total number of the interval responses divided by the total interval duration). These three rate measures showed almost the same value. Means and deviations in these measures were indistinguishable, and the statistical conclusions based on them were the same in almost all cases. Thus, we used only the rate-except-delay measure and called it the "response rate" in this and following experiments.

2. Delay rate: The cumulative number of delay responses was divided by the total delay duration.

3. Waste time per interval: The cumulative waste time was divided by the number of the interval.

4. First half ratio (FHR): The number of responses made during the first 5 segments was divided by the number of responses during all the segments. A similar measure in Gleitman and Bernheim (1963) included the response that triggered the reinforcer in the denominator, but the elimination of it seems better because it occurred after the interval. Practically, the methods produce almost the same value when the number of the responses is large, as reported here.

5. Index of curvature (IC): Calculation was based on the method of Fry, Kelleher, and Cook (1960) for the accumulated data. Constant responding gives 0 , and convergence of responding on the later segments gives a high value. With the 10 -segment interval used here, the maximum value is 0.9 , when all responses are located in the final segment. Because the FHR and the IC change systematically and reliably with changes of the shape of the response pattern during the interval (Gollub, 1964), these pattern measures were used as indices of temporal discrimination: the lower the FHR and the higher the IC, the better the temporal discrimination.

Although the pattern measures were directly used for parametric analyses, the rate measures and the waste time were subjected to a square-root transformation before the analyses. We adopted this transformation because the variances in these measures tend to increase with the mean. Such an increment was not observed in the pattern measures. In practice, however, the following statistical conclusions were almost the same whether we used the transformed or untransformed data of the rate, time, and pattern measures. In all statistical tests, the significance level was set at $p<.05$.

\section{Results}

Although the response rate was lower in Group Sig than in Group Unsig on the session of FI 15-sec, the dif- 
ference failed to reach statistical significance $[t(10)=$ $2.22]$. Other measures we investigated also did not show any reliable group difference on this session $[t \mathrm{~s}(10)<$ 2.02]. On the session of FI $30-\mathrm{sec}$, the response rate of Group Sig was significantly lower than that of Group Unsig $[t(9)=4.26]$, though the data for 1 rat in Group Unsig were lost because of a recording error. Differences between the groups in other measures were all nonsignificant $[\operatorname{ts}(9)<1]$.

The measures during the remaining sessions, in which the interval value was $60 \mathrm{sec}$, are shown in Figure 1. A split-plot analysis of variance (ANOVA) with group and session (repeated) as factors was applied to each measure.

The top panel of Figure 1 indicates the response rate. An ANOVA indicated significant group $[F(1,10)=$ $12.92]$ and session $[F(15,150)=3.09]$ effects and a nonsignificant group $\times$ session interaction $[F(15,150)=$ 1.18]. The delay rate for each group is shown in the second panel of Figure 1. An ANOVA indicated a significant group effect $[F(1,10)=55.93]$. The effect of session and the interaction were nonsignificant $[F \mathrm{~s}(15,150)<$ 1.41]. The waste time per interval is shown in the third panel of Figure 1. An ANOVA indicated significant group $[F(1,10)=5.99]$ and session $[F(15,150)=6.26]$ effects and a nonsignificant interaction $(F<1)$. The fourth panel of Figure 1 shows the FHR, and the bottom panel shows the IC. ANOVAs of these measures indicated significant session effects: the FHR $[F(15,150)=$ 43.46] and the IC $[F(15,150)=49.99]$. The group effect and the interaction were nonsignificant both in the FHR and the IC $(F s<1)$.

In summary, Group Sig responded more slowly than Group Unsig, and the waste time was greater in the former than in the latter. The waste time and the FHR decreased, and the IC increased, as training proceeded. There was no group difference in the pattern of responding, as indicated by the nonsignificant group effect and interaction in the FHR and the IC.

\section{Discussion}

We replicated the attenuating effect of the signal on the rate of FI responding using the unsignaled control instead of the yoked-signal control used by Tarpy et al. (1984). Accelerating patterns of responding during the interval, however, were the same in the signaled and unsignaled rats.

A theoretically uninteresting account of this finding is that the signal unconditionally distracted the leverpressing. For example, the rats might fear the bright light signal from the bulb located near the lever and might withdraw from the area of the bulb and the lever, resulting in slower responding. However, this is unlikely because the attenuating effect continued for 18 sessions, during which any unconditional fear should have disappeared.

The attenuation of the response rate by the signal is predicted by the account based on facilitation of temporal discrimination (Tarpy et al., 1983, 1984). However, this view makes the improper prediction of more accurate temporal patterning in the signaled group than in the unsignaled group. Although treatment of null statistical results is difficult, the temporal discrimination reflected in the pattern measures did not appear to be a causal fac-
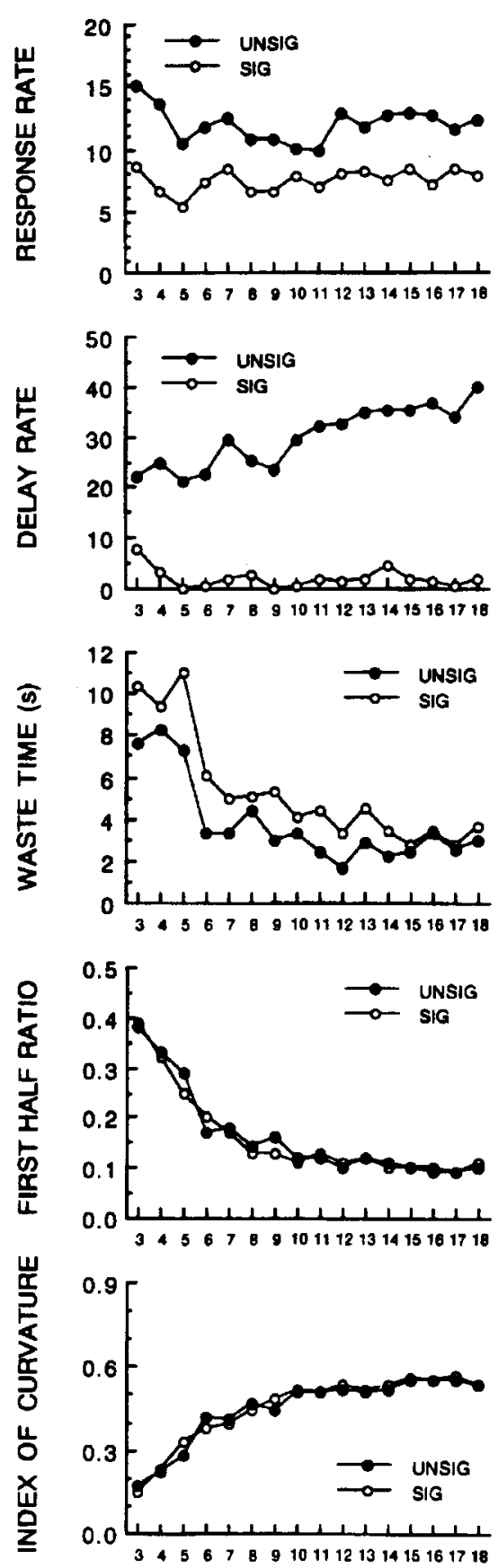

\section{SESSIONS}

Figure 1. Group mean measures of Experiment 1. Group Unsig was trained with the unsignaled delay and Group Sig received the bulb lightning signal during the delay. Top panel: the number of responses per minute except the delay period. Second panel: the number of responses per minute during the delay period. Third panel: the waste time per interval. The fourth panel: the first half ratio. Bottom panel: the index of curvature. 
tor for the significant group difference in the rate measures observed here.

According to the overshadowing hypothesis (see, e.g., Pearce \& Hall, 1978), the signaled group responds slower than the unsignaled group because the signal restricts the development of response-reinforcer association. Prediction by the overshadowing hypothesis of the pattern measures is obscure, because we have no a priori knowledge of the temporal nature of the response overshadowing.

\section{EXPERIMENT 2}

We conducted the second experiment to investigate the generality of the results of Experiment 1 . In this experiment, two kinds of signal were used. The first was the light signal as in Experiment 1, and the second was a tone signal. The light has visual and localized spatial properties, but the tone is auditory and diffuse. We compared two experimental groups, each receiving one of these signals, with an unsignaled group.

\section{Method}

Subjects and Apparatus. Eighteen experimentally naive rats were used. The sex and strain of the rats and the housing conditions were the same as those in Experiment 1. Throughout the experiment, the rats were maintained at $85 \%$ of their free-feeding weights (range, 367-427 g).

Two sets consisting of the chamber, the outer shell, and the computer were used. The first was the same as that of Experiment 1, and the second was its copy. Each chamber has an electric buzzer (National EB-2114, Japan) $3.5 \mathrm{~cm}$ below the houselight on the front panel, used to present a $500-\mathrm{msec}$ tone signal $(2300 \mathrm{~Hz}$, $100 \mathrm{~dB}$ around the lever). The two shells were in the same room, so that a masking noise from a speaker in the room, in addition to the ventilation fans' noise, was used to mitigate auditory interaction between the chambers. The noise level in each chamber was $75 \mathrm{~dB}$. Bio Serv 45-mg pellets (DPP F-200) were used as reinforcers. Experimental sessions were conducted 7 days a week, except for 1 day between Sessions 10 and 11 of Phase 1.

Procedure. Acclimation and pretraining were the same as those in Experiment 1. Then three groups of 6 rats were matched to the extent possible for performance during pretraining, the order of running on daily sessions, the chambers used, and body weight. Then all groups received an FI 15 -sec session of 60 reinforcements, an FI 30-sec sessions of 50 reinforcements, and 20 FI 60 sec sessions of 30 reinforcements (Phase 1). Each 500-msec delay of reinforcement was signaled by illumination of the light for Group Light and by the buzzer tone for Group Tone, but was not signaled for Group Unsig. After this training, all rats received the unsignaled treatment for 10 sessions when the schedule value was FI $60 \mathrm{sec}$ and the number of reinforcements was 30 (Phase 2).

Measures. The recording was in accumulated fashion except for the last session of Phase 1, where we recorded the data in the interval-by-interval manner. Analyses were based on the data accumulated throughout the experiment.

\section{Results}

Phase 1. The measures on the first (FI $15 \mathrm{sec}$ ) and the second (FI $30 \mathrm{sec}$ ) sessions were analyzed by a series of one-way completely randomized ANOVAs. On the first session of Phase 1, there was no significant group difference in any measures we investigated $[F \mathrm{~s}(2,15)<1.82]$. On the second session, there were significant group dif- ferences in the response rate $[F(2,15)=6.24]$, the delay rate $[F(2,15)=11.16]$, the waste time $[F(2,15)=3.50]$, and the FHR $[F(2,15)=4.29]$. Subsequent Fisher's least significant difference (LSD) analyses revealed the following reliable between-group differences. First, Group Light responded more slowly than Groups Unsig and Tone in the response rate and the delay rate measures. Second, Group Light showed a smaller FHR than did Group Tone. Other comparisons were all nonsignificant.

The measures during the remaining sessions, in which the interval value was $60 \mathrm{sec}$, are shown in the left portions of Figure 2. A split-plot ANOVA with group and session (repeated) as factors was applied to each measure, and LSD comparisons were used to analyze significant group effects or group $\times$ session interactions.

The top panel of Figure 2 shows the response rate. An ANOVA revealed significant group $[F(2,15)=7.70]$, session $[F(19,285)=25.03]$, and group $\times$ session $[F(38,285)=2.75]$ effects. A subsequent LSD analysis of the interaction revealed that Group Light responded more slowly than Groups Unsig and Tone throughout Phase 1, and that Group Tone responded more slowly than Group Unsig on Session 3, but more rapidly on Sessions $12,13,15,16,18,20$, and 21 .

The delay rate for each group is shown in the second panel of Figure 2. An ANOVA indicated significant group $[F(2,15)=10.26]$ and session $[F(19,285)=2.19]$ effects. The interaction was nonsignificant $(F<1)$. An LSD analysis of the group effect revealed that Group Unsig responded more rapidly than did Groups Light and Tone, whereas the latter two did not differ from each other.

The waste time per interval is shown in the third panel of Figure 2. An ANOVA showed significant group $[F(2,15)=$ $15.18]$ and session $[F(19,285)=3.88]$ effects and a nonsignificant interaction $[F(38,285)=1.09]$. An LSD analysis of the group effect revealed that Group Light wasted more time than did Groups Unsig and Tone, whereas the latter two did not differ from each other.

The fourth panel of Figure 2 shows the FHR and the bottom panel shows the IC. ANOVAs of these measures indicated significant session effects: the FHR $[F(19,285)=$ $57.26]$ and the IC $[F(19,285)=54.44]$. The group effect was significant in the IC $[F(2,15)=4.25]$ but not in the FHR $[F(2,15)=2.78]$. The interaction was not significant in either pattern measure $[F \mathbf{s}(38,285)<1.30]$. An LSD analysis of the group effect in the IC revealed that the value of Group Light was higher than those of Groups Unsig and Tone, whereas the latter two did not differ from each other.

Phase 2. The data of Phase 2 are shown in the right portion of Figure 2. The same kind of analysis used in Phase 1 was used for these data. The removal of the signal did not affect the general course of the response rate, as shown in the first panel of Figure 2. An ANOVA of the data of Phase 2 indicated a significant group effect $[F(2,15)=4.87]$. Neither the session effect $[F(9,135)=$ 1.11] nor the interaction $(F<1)$ was significant. An LSD analysis of the group effect revealed that Group Light re- 

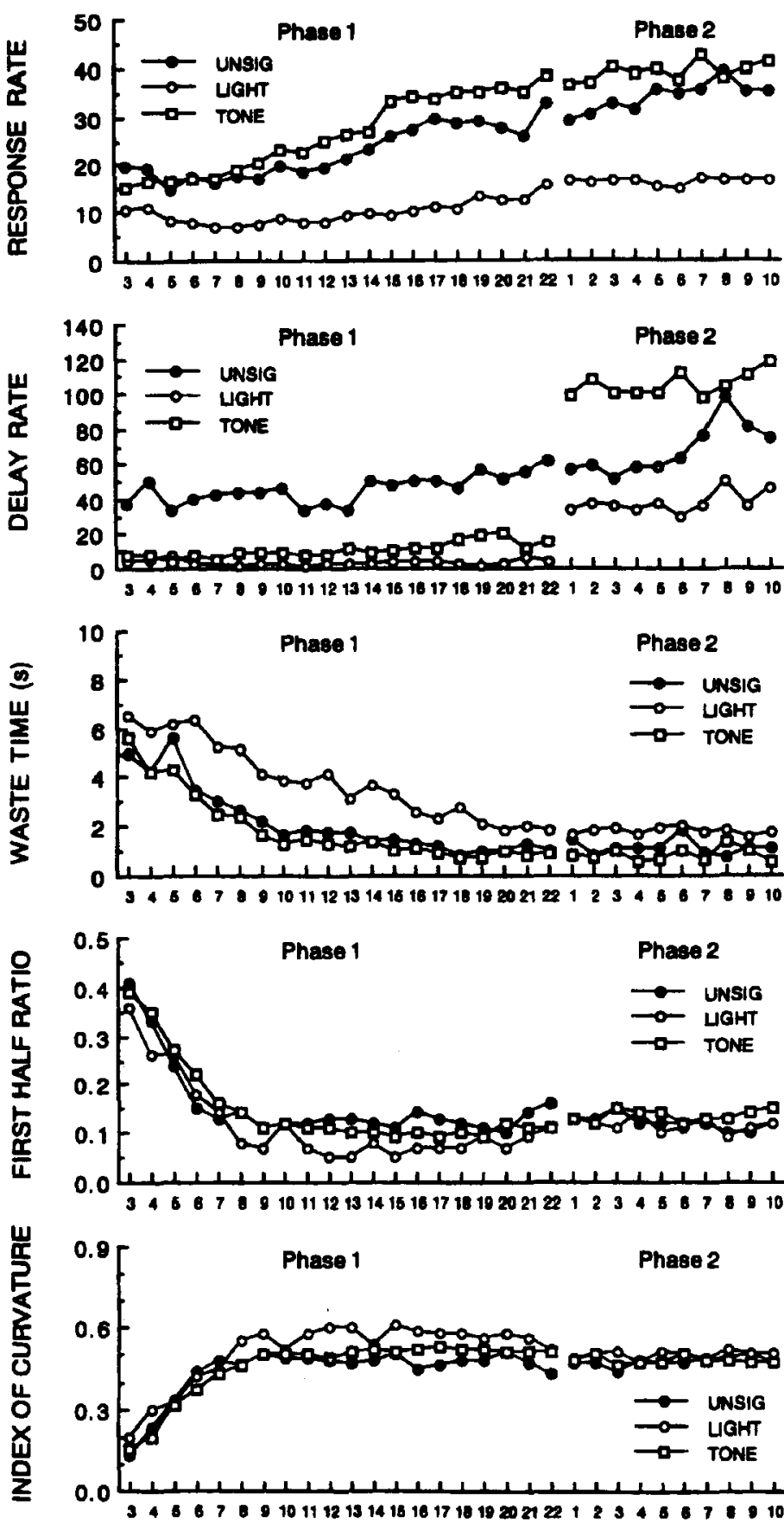

\section{SESSIONS}

Figure 2. Group mean measures of Experiment 2. Throughout Phase 1, Group Unsig was trained with the unsignaled delay, Group Light received the bulb lightning signal, and Gmup Tone received the tone signal during the delay. In Phase 2, all groups were in the unsignaled condition. Top panel: the number of responses per minute except the delay period. Second panel: the number of responses per minute during the delay period. Third panel: the waste time per interval. The fourth panel: the first half ratio. Bottom panel: the index of curvature. 
sponded more slowly than did Groups Unsig and Tone. The difference between the latter two did not reach significance.

The removal of the signals increased the delay rate in Groups Light and Tone (the second panel of Figure 2). An ANOVA of the Phase 2 data indicated significant group $[F(2,15)=5.24]$ and session $[F(9,135)=2.29]$ effects, and nonsignificant interaction $[F(18,135)=$ 1.31]. An LSD analysis of the group effect revealed a significant difference only between Groups Light and Tone.

The removal of the signal did not affect the general course of the waste time (the third panel of Figure 2). An ANOVA of the data of Phase 2 indicated a significant group effect $[F(2,15)=7.35]$. The session effect $[F(9,135)=1.05]$ and the interaction $(F<1)$ were nonsignificant. An LSD analysis of the group effect revealed that Group Light wasted more time than Groups Unsig and Tone, whereas the latter two did not differ.

There were no statistically significant differences in the pattern measures in Phase 2: ANOVAs of the FHR and the IC indicated that all $F$ values of the group and session effects and the interactions were less than 1 .

\section{Discussion}

The light signal for reinforcement attenuated response rate and facilitated temporal discrimination. These results accord with Tarpy et al.'s $(1983,1984)$ hypothesis. The facilitation of the temporal discrimination, however, is inconsistent with the results of Experiment 1 . We have no adequate explanation for this difference between experiments.

Tarpy et al.'s $(1983,1984)$ hypothesis could predict no attenuating effect of the tone, given that the tone, for instance, did not facilitate temporal discrimination. The tone might have failed to facilitate temporal discrimination because it was not sufficiently salient. Tarpy et al.'s hypothesis, however, cannot account for the enhancing effect of the tone signal on the response rate, a result predicted by the hypothesis that a signal facilitates the acquisition of a specific unit of behavior (rapid responding) preceding reinforcement. The overshadowing hypothesis (see, e.g., Pearce \& Hall, 1978) can also account for the attenuating effect of the light signal on the response rate, but cannot account for the enhancing effect of the tone signal.

The removal of the signals from two signaled groups had little effect on the response rate and the waste time, but increased the delay rate. It reduced the temporal discrimination of Group Light to the level of the other groups. The remaining group difference in the response rate between Groups Light and Unsig, without the difference in temporal discrimination between them, suggests that the facilitation of the temporal discrimination by the light signal was not the cause of the attenuation of the response rate.

It may be worthwhile to note that the rats of Experiment 2 responded more rapidly than those of Experi- ment 1 (see the top panels of Figures 1 and 2). The major reason for this might be a difference in reinforcement value of the pellets used in these experiments.

\section{EXPERIMENT 3}

To investigate the generality of the results of Experiment 2, we replicated the procedure of Experiment 2, but the FI value during the major portion of the study was $30 \mathrm{sec}$ instead of $60 \mathrm{sec}$.

\section{Method}

Subjects and Apparatus. Eighteen experimentally naive rats were used. The sex and strain of the rats and the housing conditions were the same as those in the previous experiments. Throughout the experiment, the rats were maintained at $85 \%$ of their freefeeding weights (range, $312-380 \mathrm{~g}$ ).

The chamber, the outer shell, and the computer were identical to those used in Experiment 1, with the following modifications. As in Experiment 2, the electric buzzer was used to present a 500msec tone signal and Bio Serv pellets were used as reinforcers. A Gerbrands cumulative recorder was connected with relay equipment to the lever and the dispenser. Experimental sessions were conducted 7 days a week.

Procedure. Acclimation, pretraining, and matching the rats were as in the previous experiments. Then three groups of 6 rats received an FI 15 -sec session of 60 reinforcements and 20 FI 30-sec sessions of 30 reinforcements (Phase 1). Each delay of reinforcement was signaled by illumination of the light for Group Light and by the buzzer for Group Tone; it was not signaled for Group Unsig. After this training, all rats received the unsignaled treatment for 10 sessions when the schedule value was FI $30 \mathrm{sec}$ and the number of reinforcements was 30 (Phase 2).

Measures. Although interval-by-interval recording was conducted in this experiment, the number of the responses in each interval was too small to get reliable measures. Thus we used the data accumulated over the entire session, as in the previous experiments.

\section{Results}

Phase 1. A series of one-way completely randomized ANOVAs of the session of FI $15 \mathrm{sec}$ indicated that there were no significant group effects in any measure $[F \mathrm{~s}(2,15)$ $<2.17]$ except in the delay rate $[F(2,15)=4.80]$. A Fisher's LSD analysis on the group effect of the delay rate failed to find significance in any pairwise comparison.

The measures during the remaining sessions, in which the interval value was $30 \mathrm{sec}$, are shown in the left portions of Figure 3. The same kind of analyses as in Experiment 2 were used for these data.

The top panel of Figure 3 shows the response rate. An ANOVA indicated a significant session effect $[F(19,285)$ $=14.03]$ and a group $\times$ session interaction $[F(38,285)=$ 2.75]. The group effect failed to reach significance $[F(2,15)$ $=2.59$ ]. A subsequent LSD analysis of the interaction revealed the following points. First, Group Light responded more slowly than Group Unsig on Sessions 2-5 and 7-11. Second, Group Tone responded more slowly than Group Unsig on Session 3, but more rapidly on Sessions 9, 12-14, and 16-21. Finally, Group Light responded more slowly than did Group Tone on Sessions 3 and 5-21. 

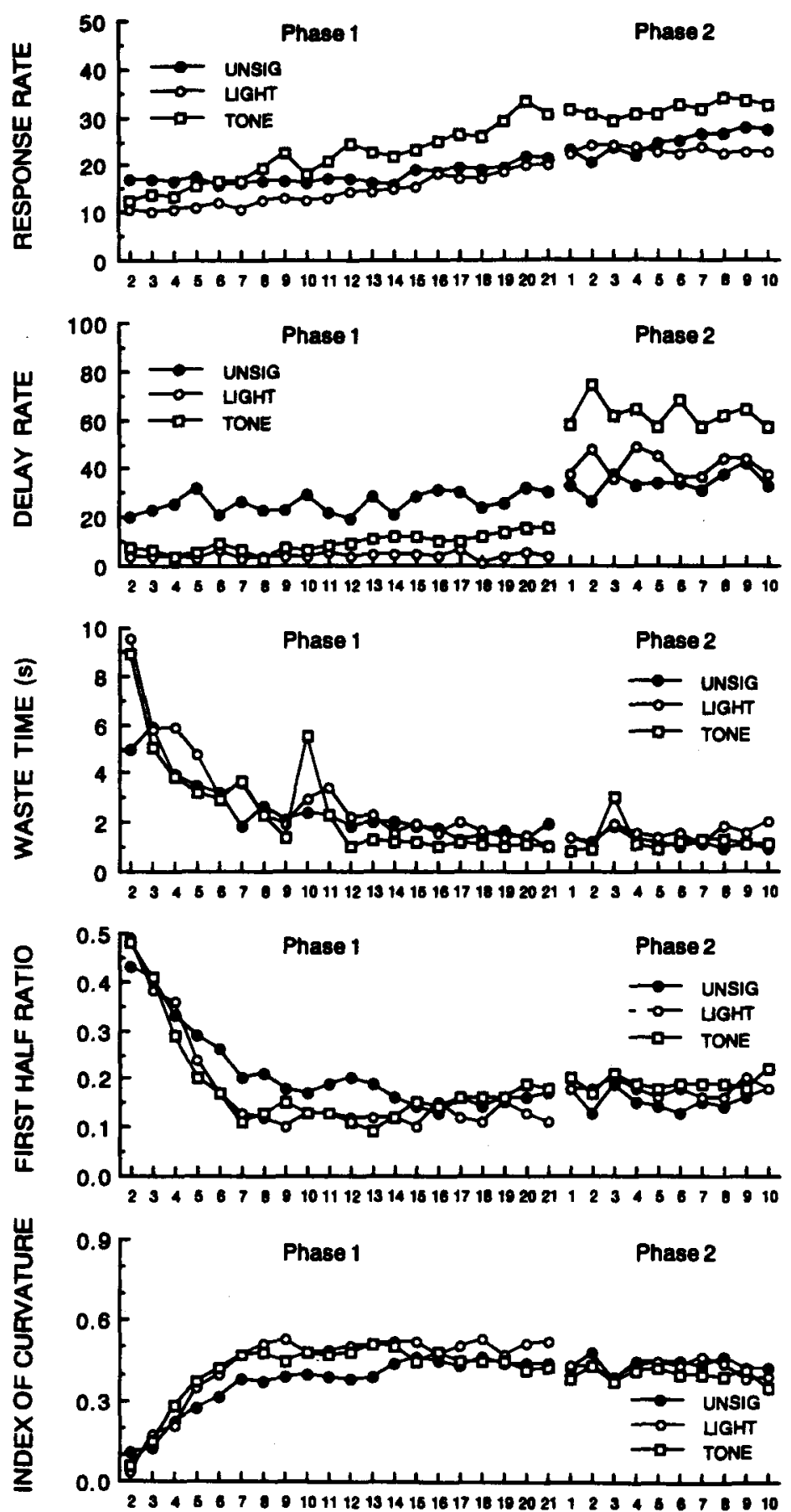

\section{SESSIONS}

Figure 3. Group mean measures of Experiment 3. Throughout Phase 1, Group Unsig was trained with the unsignaled delay, Group Light received the bulb lightning signal, and Group Tone received the tone signal during the delay. In Phase 2, all groups were in the unsignaled condition. Top panel: the number of responses per minute except the delay period. Second panel: the number of responses per minute during the delay period. Third panel: the waste time per interval. The fourth panel: the first half ratio. Bottom panel: the index of curvature. 
The delay rate for each group is shown in the second panel of Figure 3. An ANOVA indicated a significant group effect $[F(2,15)=15.07]$. The session effect and the interaction were nonsignificant $(F \mathrm{~s}<1)$. An LSD analysis of the group effect revealed that Group Unsig responded more rapidly than Groups Light and Tone, whereas the latter two did not differ from each other.

The waste time per interval is shown in the third panel of Figure 3. An ANOVA indicated a significant session effect $[F(19,285)=30.81]$ and an interaction $[F(38,285)=$ 1.62]. The group effect failed to reach significance $[F(2,15)=2.57]$. An LSD analysis of the interaction revealed the following points. First, Group Light wasted more time than did Group Unsig on Sessions 2, 4, and 7. Second, Group Tone wasted more time than did Group Unsig on Sessions 2, 7, and 10. Finally, Group Light wasted more time than did Group Tone on Sessions 4 and 12.

The fourth panel of Figure 3 shows the FHR, and the bottom panel shows the IC. ANOVAs of these measures indicated significant session effects: the FHR $[F(19,285)=$ $53.00]$ and the IC $[F(19,285)=56.84]$. The group effect was not significant for either measure: the FHR $[F(2,15)=1.35]$ and the IC $[F(2,15)=1.83]$. The interaction was significant in both measures: the FHR $[F(38,285)=1.67]$ and the $\operatorname{IC}[F(38,285)=1.73]$.

An LSD analysis of the interaction in the FHR revealed the following points. First, Group Light showed lower values than Group Unsig on Sessions 6-9, 12, and 13. Second, Group Tone showed lower values than Group Unsig on Sessions 5-8 and 11-13. Finally, Group Light showed lower values than Group Tone on Sessions 4 and 21. An LSD analysis of the interaction in the IC revealed similar results. First, Group Light showed larger values than Group Unsig on Sessions 2, 4-13, and 21. Second, Group Tone showed larger values than Group Unsig on Sessions 5-8 and 10-13. Finally, Group Light showed larger values than Group Tone on Sessions 9, 18, 20 , and 21 . These analyses suggested that both signals facilitated the temporal discrimination and that the effect of the light signal was larger than that of the tone.

Phase 2. The data of Phase 2 are shown in the right portion of Figure 3. The same kind of analyses as in Phase 1 were used for these data. There were no significant differences among the rate measures. An ANOVA of the response rate of Phase 2 (the top panel of Figure 3) indicated nonsignificant group $(F<1)$ and session $[F(9,135)=1.70]$ effects and a nonsignificant interaction $[F(18,135)=1.16]$. An ANOVA of the delay rate (the second panel of Figure 3 ) indicated nonsignificant group $[F(2,15)=2.12]$ and session $(F<1)$ effects and interaction $(F<1)$.

An ANOVA of the waste time (the third panel of Figure 3 ) revealed a nonsignificant group effect $[F(2,15)=$ $1.34]$ and interaction $(F<1)$. The session effect, however, was significant $[F(9,135)=2.09]$.

Although the effect of session failed to reach significance in an ANOVA of the FHR $[F(9,135)=1.93]$, it was significant in an ANOVA of the IC $[F(9,135)=2.79]$.
The group effect and the interaction were nonsignificant in both the measures $(F \mathrm{~S}<1)$.

\section{Discussion}

The light signal for the reinforcement attenuated the response rate, though this effect became smaller in the later sessions of Phase 1 . In addition, it facilitated the temporal discrimination throughout Phase 1 . These results seem to accord with the hypothesis of Tarpy et al. $(1983,1984)$, as well as with the results of Experiment 2. Their hypothesis, however, would predict low responding with the tone signal because it facilitated the temporal discrimination in this experiment. Although the tone signal attenuated the response rate during the third session, it enhanced the response rate during later sessions. The attenuating effect during the early sessions was also observed in Experiment 2, so it might have been produced by unconditional properties of the tone.

The overshadowing hypothesis (see, e.g., Pearce \& Hall, 1978) also might account for the attenuating effect of the light signal on the response rate. It might be worthwhile to note that the weakening of this effect with extended training corresponds to a similar finding in a stimulusstimulus overshadowing study (Bellingham \& Gillette, 1981). However, the overshadowing hypothesis does not account for the enhancing effect of the tone signal any better than Tarpy et al.'s $(1983,1984)$ hypothesis. An adequate account of the enhancing effect appears to require that the signal facilitate a unit of behavior preceding reinforcement. If the tone signal facilitates the rapid responding of the later portion of the fixed interval, the response rate should be higher in the tone-signaled group than in the unsignaled group.

Upon removal of the signals from two signaled groups, the delay rate increased and the better temporal discrimination of Group Light than Groups Unsig and Tone disappeared. Though Figure 3 suggests that the removal of the signals had little impact on the response rate, the group differences found before removal of the signal were not found afterward.

\section{GENERAL DISCUSSION}

According to Tarpy et al. $(1983,1984)$, a signal for reinforcement attenuates instrumental responding maintained on FI schedules because it facilitates temporal discrimination. Indeed, a localized light signal attenuated leverpressing and facilitated the temporal discrimination in Experiments 2 and 3 in the present study. However, it attenuated the response rate without facilitating the temporal discrimination in Experiment 1. In addition, removal of the light signal eliminated the better temporal discrimination in the light-signaled group, but the attenuated responding did not recover in this group after the removal of the signal (Experiment 2). These results suggest that facilitation of temporal discrimination is not a necessary condition for attenuation of responding. Furthermore, in Experiment 3, a diffuse tone signal facilitated the temporal discrimination, but enhanced the 
response rate. Thus, facilitation of temporal discrimination is not a sufficient condition for attenuation of responding.

The overshadowing hypothesis (see, e.g., Pearce \& Hall, 1978) also fails to account for response enhancement by the tone signal in Experiments 2 and 3, because it predicts response attenuation by any signal that is associated with reinforcement. One cannot argue the possibility that the tone signal was too faint to acquire enough association with the reinforcement and to attenuate the responding, because it had the opposite effect rather than no effect on responding.

Studies of signaled reinforcement effects have yielded three other hypotheses that we have not yet mentioned. Two of them, an efficiency hypothesis (Roberts, Tarpy, \& Lea, 1984; Tarpy \& Roberts, 1985) and a burst hypothesis (Lattal \& Ziegler, 1982; Richards, 1981; Williams, 1982; Williams \& Heyneman, 1982), also cannot account for the finding that the effects of the signal depended on its nature: the light or the tone.

A third alternative account, the tracking hypothesis, is based on observing (Iversen, 1981), orienting (Williams, 1982; Williams \& Heyneman, 1982), or tracking (Reed, 1992; Roberts, Tarpy, \& Cooney, 1985) behavior to a signal location. According to this hypothesis, pairing of the signal with the food elicits the behavior directed at the signal location and thus attenuates instrumental responding. The attenuation of the instrumental responding can originate from at least three sources.

First, the tracking behavior might occur not only during the signal period but also during the interreinforcement interval, so that it peripherally competes with the ongoing instrumental responding. Second, the tracking behavior elicited by the signal might be associated with the reinforcer and thus might restrict the development of an instrumental response-reinforcer association. This is responseresponse overshadowing rather than the stimulus-response overshadowing that we have considered. Finally, a sequence of the instrumental response followed by the elicited tracking behavior might be strengthened by superstitious reinforcement (Herrnstein, 1966). In our case, press-and-track sequential behavior might have resulted in a lower pressing rate during the interval than simple pressing behavior.

The tracking hypothesis seems to suit best the different effects of the light and tone signals. The localized nature of the light signal tends to elicit tracking behavior, resulting in the attenuation of the instrumental leverpressing. On the other hand, elicitation of tracking behavior by the tone signal would be small because of its diffuse nature. Consequently, we could observe the enhancing effect of the reinforcement signal in the tonesignaled group (see Reed et al., 1988b).

According to Reed and his colleagues (e.g., Reed, 1989a; Reed \& Hall, 1988), the signal facilitated the unit of behavior preceding reinforcement. In this article, we have treated the rapid responding in the later portion of the fixed interval as the unit of behavior to be facilitated. This specification accords with our finding of the en- hancing effect of the tone signal on the response rate. However, on FI schedules the required interval is more likely to end during a longer interresponse time (IRT) (Dews, 1969). Thus, if we treat this long IRT as the unit of behavior to be facilitated, the signal should attenuate, rather than enhance, the rate of responding. In addition, Schachtman and Reed (1992) presented an experiment in which a two-response sequence was reinforced, and the signal facilitated learning about the initial, rather than the terminal, response. If the signal facilitates the initial portion of the fixed interval, the response rate will be reduced. Therefore, we need some way to specify what kind of behavior unit is to be facilitated by the signal. Without this independent specification, the choice of unit can be based on the effects obtained.

The different effects of the signals reported in the present study may be explained by an account based on the facilitation of the rapid responding preceding the reinforcement by the tone signal and tracking behavior elicited by the light signal. However, this account, as well as the other hypotheses considered, cannot explain the effects of the signals on the temporal response patterning. Furthermore, the effects of the signals on the response rate were not reversible. The removal of the light signal did not enhance the low response rate attained in the light-signaled condition, and the removal of the tone signal did not attenuate the high response rate attained in the tone-signaled condition. Thus the account described above is adequate only for acquisition, and we need another account for the persistence of acquired responding.

FI schedules have been shown to induce many nonreinforced behaviors (see, e.g., Anderson \& Shettleworth, 1977; Roper, 1978; Staddon \& Simmelhag, 1971), and the overall frequency and the temporal pattern of the reinforced behavior seems to be affected by the induced behaviors (Osborne, 1978; Staddon, 1977). Information on other behaviors occuring will provide empirical support for the tracking hypothesis and may explain two effects of the signals: the irreversibility of the effect on the response rate and the facilitation of the temporal discrimination observed in some conditions. In addition, further research with more finely grained analyses of the responding, such as IRT analysis (e.g., Gentry, Weiss, \& Laties, 1983; Palya, 1992; Shull \& Brownstein, 1970; Staddon \& Frank, 1975), might shed more light on the process of the complex signal effects observed here.

\section{REFERENCES}

Anderson, M. C., \& Shettleworth, S. J. (1977). Behavioral adaptation to fixed-interval and fixed-time food delivery in golden hamsters. Journal of the Experimental Analysis of Behavior, 25, 33-49. Bellingham, W. P., \& Gillette, K. (1981). Attenuation of overshadowing as a function of nondifferential compound conditioning trials. Bulletin of the Psychonomic Society, 18, 218-220.

Dews, P. B. (1969). Studies on responding under fixed-interval schedules of reinforcement: The effects on the pattern of responding of changes in requirement at reinforcement. Journal of the Experimental Analysis of Behavior, 12, $191-199$.

DEws, P. B. (1978). Studies on responding under fixed-interval schedules of reinforcement: II. The scalloped pattern of the cumulative record. Journal of the Experimental Analysis of Behavior, 29, 67-75. 
Dickinson, A., Peters, R. C., \& Shechter, S. (1984). Overshadowing of responding on ratio and interval schedules by an independent predictor of reinforcement. Behavioural Processes, 9, 421-429.

ECKerman, D. A., \& MCGourTy, D. P. (1969). Varying temporal placement of response-produced stimuli in a fixed-interval schedule. Psychonomic Science, 15, 225-227.

FARMER, J., \& SChOENFELD, W. N. (1966a). The effect of a responsecontingent stimulus introduced into a fixed-interval schedule at varying temporal placement. Psychonomic Science, 6, 15-16.

FARMER, J., \& Schoenfeld, W. N. (1966b). Varying temporal placement of an added stimulus in a fixed-interval schedule. Journal of the Experimental Analysis of Behavior, 9, 369-375.

FERSTER, C. B., \& SKINNER, B. F. (1957). Schedules of reinforcement. New York: Appleton.

Fry, W., Kelleher, R. T., \& CoOK, L. (1960). A mathematical index of performance on fixed-interval schedules of reinforcement. Journal of the Experimental Analysis of Behavior, 3, 193-199.

Gentry, G. D., Weiss, B., \& Laties, V. G. (1983). The microanalysis of fixed-interval responding. Journal of the Experimental Analysis of Behavior, 39, 327-343.

GleitMan, H., \& BeRnHEIM, J. W. (1963). Retention of fixed-interval performance in rats. Journal of Comparative \& Physiological Psychology, 56, 839-841.

GolluB, L. R. (1964). The relations among measures of performance on fixed-interval schedules. Journal of the Experimental Analysis of Behavior, 7, 337-343.

HALL, G. (1982). Effects of a brief stimulus accompanying reinforcement on instrumental responding in pigeons. Learning \& Motivation, 13, 26-43.

Hall, G., Channell, S., \& Pearce, J. M. (1981). The effects of a signal for free or earned reward: Implications for the role of responsereinforcer associations in instrumental performance. Quarterly Journal of Experimental Psychology, 33B, 95-107.

HERRNSTEIN, R. J. (1966). Superstition: A corollary of the principles of operant conditioning. In W. K. Honig (Ed.), Operant behavior: Areas of research and application (pp. 33-51). New York: Appleton.

IVERSEN, I. H. (1981). Response interactions with signaled delay of reinforcement. Behaviour Analysis Letters, 1, 3-9.

KENDALl, S. B. (1972). Some effects of response-dependent clock stimuli in a fixed-interval schedule. Journal of the Experimental Analysis of Behavior, 17, 161-168.

LatTal, K. A., \& Ziegler, D. R. (1982). Briefly delayed reinforcement: An interresponse time analysis. Journal of the Experimental Analysis of Behavior, 37, 407-416.

OSBORNE, S. R. (1978). A quantitative analysis of the effects of amount of reinforcement on two response classes. Journal of Experimental Psychology: Animal Behavior Processes, 4, 297-317.

Palya, W. L. (1992). Dynamics in the fine structure of schedulecontrolled behavior. Journal of the Experimental Analysis of Behavior, 57, 267-287.

Pearce, J. M., \& Hall, G. (1978). Overshadowing the instrumental conditioning of a lever-press response by a more valid predictor of the reinforcer. Journal of Experimental Psychology: Animal Behavior Processes, 4, 356-367.

REED, P. (1989a). Influence of interresponse time reinforcement on signalled-reward effect. Journal of Experimental Psychology: Animal Behavior Processes, 15, 224-231.

REED, P. (1989b). Marking effects in instrumental performance on DRH schedules. Quarterly Journal of Experimental Psychology, 41 B, 337-353.

REED, P. (1992). Signalled delay of reward: Overshadowing versus sign-tracking explanations. Learning \& Motivation, 23, 27-42.

ReED, P., \& HALL, G. (1988). The schedule dependency of the signaled reinforcement effect. Learning \& Motivation, 19, 387-407.

ReED, P., \& HaLL, G. (1989). The quasi-reinforcement effect: The influence of brief stimuli uncorrelated with reinforcement on variable ratio schedules. Learning \& Motivation, 20, 242-261.

ReEd, P., Schachtman, T. R., \& Hall, G. (1988a). Overshadowing and potentiation of instrumental responding in rats as a function of the schedule of reinforcement. Learning \& Motivation, 19, 13-30.

Reed, P., Schachtman, T. R., \& Hall, G. (1988b). Potentiation of responding on a VR schedule by a stimulus correlated with reinforce- ment: Effects of diffuse and localized signals. Animal Learning \& Behavior, 16, 75-82.

Reed, P., Schachtman, T. R., \& Hall, G. (1991). Effect of signaled reinforcement on the formation of behavioral units. Journal of Experimental Psychology: Animal Behavior Processes, 17, 475-485.

Reed, P., Schachtman, T. R., \& Rawlins, J. N. P. (1992). The effect of signalled reinforcement on a synthetic VI schedule. Learning \& Motivation, 23, 170-182.

Richards, R. W. (1981). A comparison of signaled and unsignaled delay of reinforcement. Journal of the Experimental Analysis of Behavior, 35, 145-152.

Roberts, J. E., TARPY, R. M., \& CoOney, N. (1985). The effects of signaled reward on sign tracking response rate, Animal Learning \& Behavior, 13, 13-17.

Roberts, J. E., TARPy, R. M., \& LEA, S. E. G. (1984). Stimulusresponse overshadowing: Effects of signaled reward on instrumental responding as measured by response rate and resistance to change. Journal of Experimental Psychology: Animal Behavior Processes, 10, 244-255.

ROPER, T. J. (1978). Diversity and substitutability of adjunctive activities under fixed-interval schedules of food reinforcement. Journal of the Experimental Analysis of Behavior, 30, 83-96.

St. Claire-Smith, R. (1979). The overshadowing of instrumental conditioning by a stimulus that predicts reinforcement better than the response. Animal Learning \& Behavior, 7, 224-228.

ST. ClaIRE-SMITH, R. (1987). Interaction of the effects of overshadowing and reinforcer devaluation manipulations on instrumental performance. Learning \& Motivation, 18, 167-184.

Schachtman, T. R., \& ReED, P. (1990). The role of response-reinforcer correlation in signaled reinforcement effects. Animal Learning \& Behavior, 18, 51-58.

Schachtman, T. R., \& Reed, P. (1992). Reinforcement signals facilitate learning about early behaviors of a response sequence. Behavioural Processes, 26, 1-11.

SCHNEIDER, B. A. (1969). A two-state analysis of fixed-interval responding in the pigeon. Journal of the Experimental Analysis of Behavior, 12, 677-687.

SEGAL, E. F. (1962). Exteroceptive control of fixed-interval responding. Journal of the Experimental Analysis of Behavior, 5, 49-57.

Shuld, R. L., \& Brownstein, A. J. (1970). Interresponse time duration in fixed-interval schedules of reinforcement: Control by ordinal position and time since reinforcement. Journal of the Experimental Analysis of Behavior, 14, 49-53.

Staddon, J. E. R. (1977). Schedule-induced behavior. In W. K. Honig \& J. E. R. Staddon (Eds.), Handbook of operant behavior (pp. 125152). Englewood Cliffs, NJ: Prentice-Hall.

Staddon, J. E. R., \& Frank, J. A. (1975). Temporal control on periodic schedules: Fine structure. Bulletin of the Psychonomic Society, 6, 536-538

Staddon, J. E. R., \& SimmelhaG, V. L. (1971). The "superstition" experiment: A reexamination of its implications for the principles of adaptive behavior. Psychological Review, 78, 3-43.

TARPY, R. M., LeA, S. E. G., \& Midgley, M. (1983). The role of response-reward correlation in stimulus-response overshadowing. Quarterly Journal of Experimental Psychology, 35B, 53-65.

TARPY, R. M., \& RoBERTS, J. E. (1985). Effects of signaled reward in instrumental conditioning: Enhanced learning on DRL and DRH schedules of reinforcement. Animal Learning \& Behavior, 13, 6-12.

TARPY, R. M., Roberts, J. E., LeA, S. E. G., \& Midgley, M. (1984). The stimulus-response overshadowing phenomenon with VI versus FI schedules of reinforcement. Animal Learning \& Behavior, 12, 50-54.

Williams, B. A. (1982). Blocking the response-reinforcer association. In M. L. Commons, R. J. Herrnstein, \& A. R. Wagner (Eds.), Quantitative analyses of behavior: Vol. 3. Acquisition (pp. 427-447). Cambridge, MA: Ballinger.

Williams, B. A., \& Heyneman, N. (1982). Multiple determinants of "blocking" effects on operant behavior. Animal Learning \& Behavior, 10, 72-76.

(Manuscript received March 17, 1994; revision accepted for publication May 24, 1995.) 